\title{
EGD Assisted Stapled Strictureplasty for Gastric Stricture after GIST Tumor Resection: A Case Report
}

\author{
Anna Goldenberg ${ }^{*}$, Roy Sandau², Adeshola Fakulujo ${ }^{3}$ \\ ${ }^{1}$ Department of Surgery, Rowan University SOM Kennedy University Hospital, Stratford, USA \\ ${ }^{2}$ General Surgery-Advocare, Rowan University SOM Kennedy University Hospital, \\ Cherry Hill, USA \\ ${ }^{3}$ Minimally Invasive Surgery Kennedy University Hospital, Rowan University SOM Kennedy University Hospital, \\ Cherry Hill, USA \\ Email: *agolde5044@yahoo.com
}

Received 15 February 2014; revised 15 March 2014; accepted 22 March 2014

Copyright (C) 2014 by authors and Scientific Research Publishing Inc.

This work is licensed under the Creative Commons Attribution International License (CC BY).

http://creativecommons.org/licenses/by/4.0/

(c) (i) Open Access

\section{Abstract}

A stricture formation resulting in impedance of gastric emptying is a fairly common complication after gastric surgery with endoscopy being the most direct way of diagnosis. When this and other methods fail in detecting the complication, the proper diagnosis can become challenging. We present an unusual case of an acquired gastric stricture after the gastrointestinal stromal tumor (GIST) resection. A stapled strictureplasty was completed with the simultaneous application of the diagnostic laparoscopy and the intra-operative EGD. We perform the comprehensive literature review (Pubmed-Cochrane 1990-current) diagnostic and surgical treatments of post resection gastric strictures.

\section{Keywords}

Gastrointestinal Stromal Tumor, Gastric Stricture, Minimally Invasive Surgery, EGD, Laparoscopy

\section{Case Report}

The patient is a 66-year-old female with complaints of chronic abdominal epigastric pain and early satiety. She had a wedge resection for GIST tumor 16 months prior after which she began experiencing abdominal pain. After numerous EGDs and multiple CT scans, no obvious reason for the pain was discovered. The patient also had

${ }^{*}$ Corresponding author. 
a small umbilical hernia.

The decision was made to perform a diagnostic laparoscopy along with intra-operative EGD repairing the umbilical hernia afterwards as its site was intended to be used for one of the port sites.

The patient was taken to the operating room, administered general endotracheal anesthesia, placed in the lithotomy position. We used Hassan port technique for entry into the abdomen. A 3-cm supra-umbilical incision was made with the scalpel at the site of the previous incision and its scar. This site was previously used at the time of GIST tumor resection for the initial diagnostic laparoscopy. We then realized that it was incisional hernia from prior port site that has presented as an umbilical hernia prior to surgery.

Once the entry into the abdomen cavity was obtained, the abdomen was insufflated with $\mathrm{CO}_{2}$. The initial laparoscopy revealed multiple adhesions between the stomach, liver, anterior abdominal wall, and the loops of the small and large bowel. We were able to place two more 5-mm trocars under direct vision: one in the left upper quadrant and the other slightly above the level of the umbilicus in the midclavicular line on left. We undertook very extensive lysis of adhesions using endoscopic scissors or the harmonic scalpel as appropriate, removing adhesions between the stomach and the liver (see Figure 1), and the stomach and the abdominal wall. It also looked like the duodenum which was adhered all the way to the lateral abdominal wall likely causing angulation at that point which could possibly be the reason of patient's symptoms; therefore the adhesions of the duodenum to the lateral abdominal wall were also released.

During the intra-operative EGD, we reached what thought to be pylorus (see Figure 2). Yet we noticed that the light from the EGD scope was seen in the body of the stomach, not near the pylorus. Further inflation of the stomach allowed us to discover the gastric stricture at the old gastric staple line (Figure 3, Figure 4). The indicated area in Figure 4 is a pylorus. The decision was made to proceed with strictureplasty. We exchanged the two 5-mm ports for 15 and 12-mm in order to accommodate the Endo GIA stapler and placed 1 additional 5-mm port to the right and slightly above the umbilicus to aid in retraction. We proceeded with the release of scar tissue going up on the greater curvature of the stomach towards the short gastrics until we had enough length of the freed up stomach for strictureplasty. The opening was made on the stomach at the stricture using the harmonic scalpel and the Covidien stapler with the green refill was placed inside. The graspers were used to aid in the positioning of the stomach and the stapler was fired in such a way that the lesser curvature of the stomach was brought together to get stapled and divided to increase the diameter of the stricture. After that, a 2-0 Vicryl suture was used endoscopically to close the opening in the transverse direction with two layers of the sutures.

The following repeat EGD with simultaneous laparoscopy showed wide patent gastric lumen (Figure 5). After the successful bubble leak test and the JP drain placement, we proceeded with the closure of the port sites along with the incisional hernia.

The patient tolerated the procedure well and there were no complications. Postoperative course was uncomplicated. We did obtain UGI on postoperative day number 1 that showed patent gastric lumen with no contrast extravasation. The patient's diet was advanced and she was discharged home on postoperative day number 3.

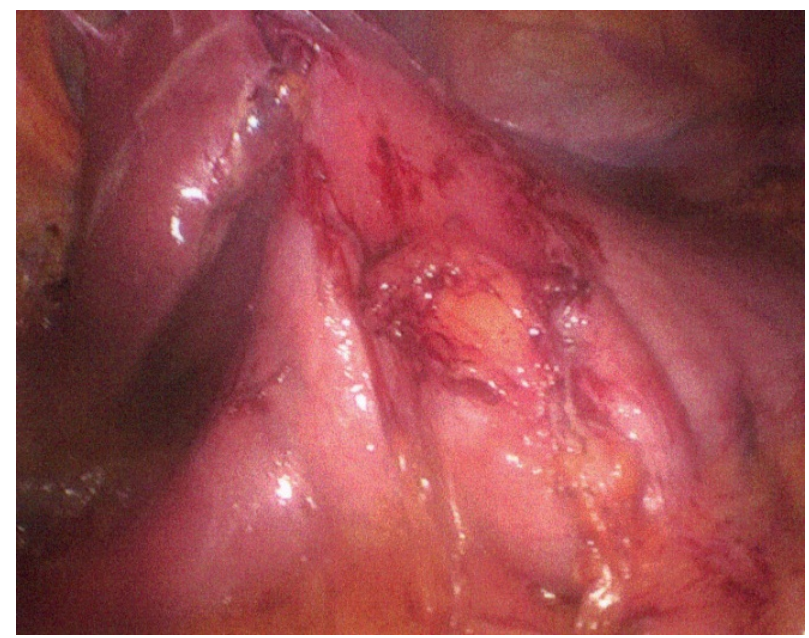

Figure 1. Adhesions between the stomach and the liver. 


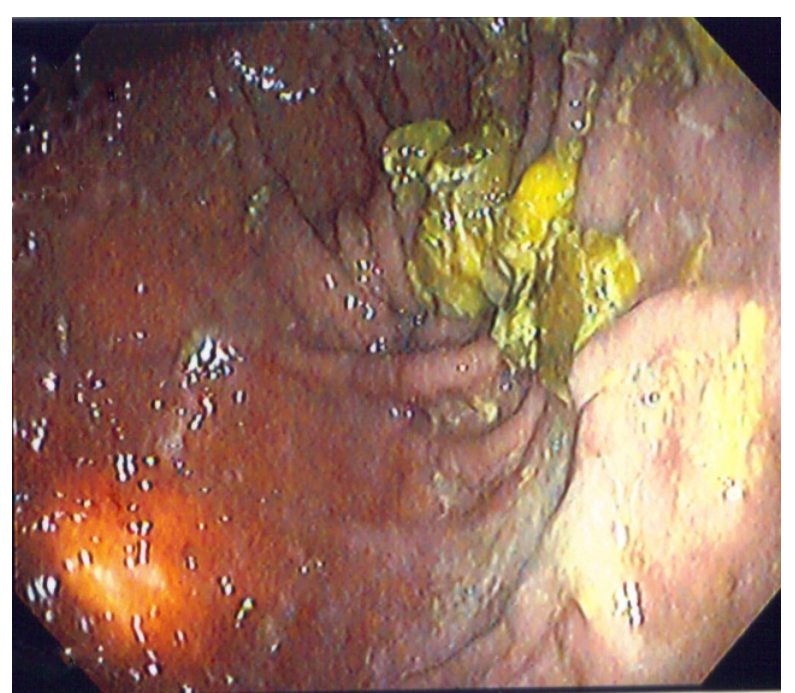

Figure 2. EGD image of the structure.

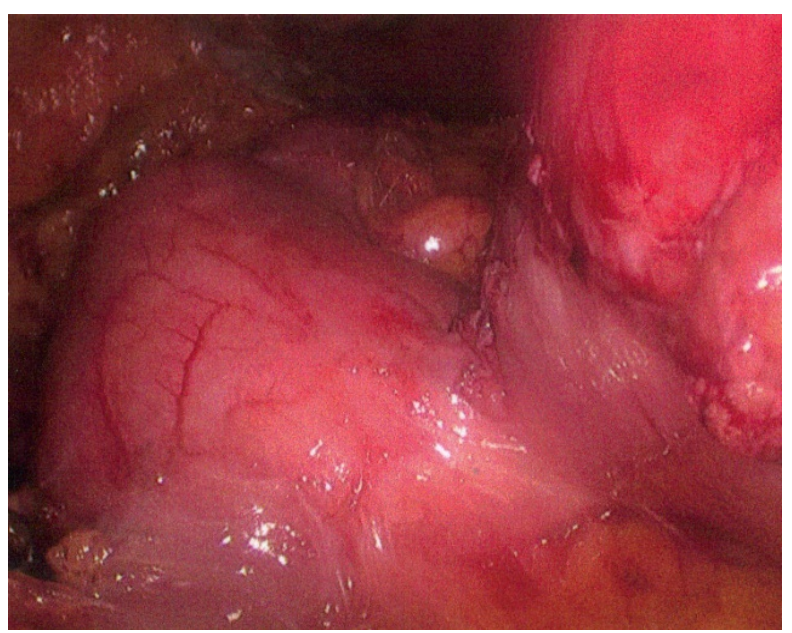

Figure 3. Insufflated stomach with stricture at the old gastric staple line.

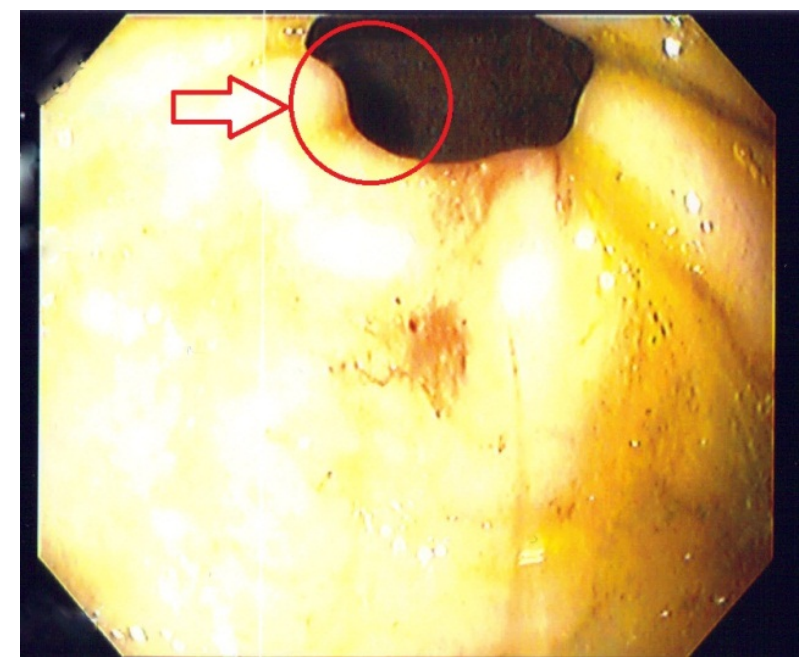

Figure 4. EGD of the stricture with the pylorus in the background. 


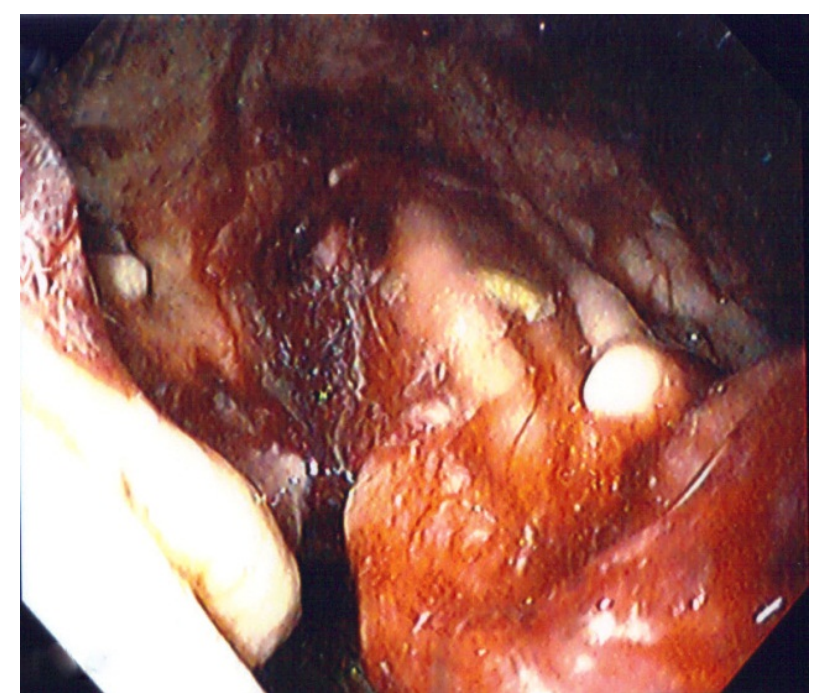

Figure 5. EGD with simultaneous laparoscopy showing a wide patent lumen post strictureplasty.

Two weeks later patient was seen for follow up in the office and admitted to some improvement in her abdominal pain as well as being able to tolerate larger meals.

\section{Discussion}

Stricture formations resulting in impedance of gastric emptying are fairly common complications after gastric surgeries and are most commonly described in the literature after bariatric surgical procedures where they occur most often. This complication usually presents within 3 to 13 weeks after surgery [1] and is manifested by progressive intolerance to solid food and then to liquid food intake. The symptoms include epigastric pain, early satiety, nausea, and vomiting.

Our patient was first readmitted with epigastric pain 4 weeks after the GIST recession. The EGD examination did not reveal any abnormalities and the balloon dilator that was passed through the narrowed area in the region of surgical changes was blown up to $20 \mathrm{~mm}$. The next several EGD along with abdominal CT with contrast showed no obstruction.

It has been established that endoscopy is the most straightforward method of making the stricture diagnosis. Yet in our case neither EGD nor exploratory laparoscopy along could discover the complication, only simultaneous application of both allowed us to successfully diagnose the stricture.

While the therapeutic options for gastric strictures include balloon dilation as the most popular and widely used [2] [3], there is evidence that the recurrence rate of this complication increase over time and the surgical revision may be required [4]. In our case we decided that a surgical procedure was more appropriate than balloon dilatation. Once the stricture was diagnosed the procedure was performed safely and quickly.

\section{Conclusion}

Stricture formation is a fairly common complication after gastric surgery and easily diagnosed with the endoscope being the most straightforward method. When this and other methods fail in detecting the complication, the simultaneous use of diagnostic laparoscopy and intraoperative EGD should be considered.

\section{References}

[1] Holt, P.D., de Lange, E.E. and Shaffer, Jr., H.A. (1995) Stricture after Gastric Surgery: Treatment with Fluoroscopically Guided Balloon Dilatation. American Journal of Roentgenology, 164, 895-899. http://dx.doi.org/10.2214/ajr.164.4.7726043

[2] Vance, P.L., de Lange, E.E., Shaffer, H.A. and Schirmer, B. (2002) Gastric Outlet Obstruction Following Surgery for Morbid Obesity: Efficacy of Fluoroscopically Guided Balloon Dilation. Radiology, 222, 70-72. 
http://dx.doi.org/10.1148/radiol.2221010640

[3] Keiichi, U., Kohhei, O., Mikihiro, I. and Masato, K. (2005) Stapled Gastroplasty for Antral Stricture after Repair of Neonatal Gastric Rupture: Report of a Case. Pediatric Surgery International, 21, 331-332. http://dx.doi.org/10.1007/s00383-004-1360-y

[4] Henry, B., George, S.M.C. and Walter, J.P. (2007) Surgical Management of Obesity. Saunders Elsevier, Philadelphia, 303. 